\title{
Groin pain associated with ultrasound finding of inguinal canal posterior wall deficiency in Australian Rules footballers
}

\author{
J W Orchard, J W Read, J Neophyton, D Garlick
}

\begin{abstract}
Objectives-To investigate the prevalence of inguinal canal posterior wall deficiency (sports hernia) in professional Australian Rules footballers using an ultrasound technique and correlate the results with the clinical symptom of groin pain.

Methods-Thirty five professional Australian footballers with and without groin pain were investigated blind with a dynamic high resolution ultrasound technique for presence of posterior wall deficiency.

Results-Fourteen players had a history of significant recent groin pain and ten of these were found to have bilateral inguinal canal posterior wall deficiency $(p<0.01)$. The relative risk for a history of groin pain with bilateral deficiency was 8.0 (95\% confidence interval 1.73 to 37.1 ). Groin pain was also found to be associated with increasing age $(p<0.01)$ which was an independent risk factor. Surgical, clinical, and ultrasound follow up for players who underwent hernia repair confirmed the validity of ultrasound as a diagnostic tool. Conclusions-Dynamic ultrasound examination is able to detect inguinal canal posterior wall deficiency in young males with no clinical signs of hernia. This condition is very prevalent in professional Australian Rules footballers, including some who are asymptomatic. There was a correlation between bilateral deficiency and groin pain, although the temporal relationship between the clinical and ultrasound findings is not established by the current study. Ultrasound shows promise as a diagnostic tool in athletes with chronic groin pain who are considered possible candidates for hernia repair. (Br f Sports Med 1998;32:134-139)
\end{abstract}

Keywords: diagnostic ultrasound; football injuries; groin pain; inguinal canal; sports hernia

Injuries to the groin region are common in athletes, particularly in the various football codes, including Australian football, ${ }^{1}$ soccer, ${ }^{2}$ and rugby league. ${ }^{3}$ The diagnosis and management of chronic groin pain in athletes is difficult and controversial. Reviews of the differential diagnosis of groin pain list multiple causes. $^{4-8}$ The diagnoses reported as being most common are adductor tendon lesions, osteitis pubis, and inguinal canal lesions (also known as incipient hernia, sports hernia, ${ }^{9}$ con- joined tendon lesions, ${ }^{10}$ pubalgia, ${ }^{11}$ and Gilmore's groin ${ }^{12}$ ). Osteitis pubis has well defined radiological diagnostic criteria, but a poor prognosis with suggested conservative management. ${ }^{13}$ Inguinal canal lesions, on the other hand, are successfully diagnosed by finding a deficient posterior inguinal canal wall at surgery and treated by hernia repair, but lack definitive criteria for presurgical diagnosis. ${ }^{14} 15$

Intraperitoneal contrast herniography has been used to show protrusions through the inguinal canal in patients with chronic groin pain. ${ }^{16}{ }^{17}$ The findings are neither fully sensitive nor specific, ${ }^{7}$ and it is an invasive procedure with a risk of complications. ${ }^{18}{ }^{19}$ Ultrasound has been reported recently as an aid in the diagnosis of hernia in non-athletes, ${ }^{19-23}$ and in our hands has been studied in the athletic population for four years.

Australian football (also known as Australian Rules football) is a code that involves progression of an oval shaped ball on a large size ground mainly using punt kicking. It is most similar to Gaelic football, but shares with soccer the repetitive changing of direction and kicking that are thought to make groin injuries such common presentations in these sports.

The aim of this study was to use a dynamic ultrasound technique to diagnose posterior inguinal canal deficiency in a population of Australian footballers (both symptomatic and asymptomatic for groin pain).

\section{Methods}

Thirty six players on the 1995 training list of the Sydney Swans Australian football team volunteered for the study. One player had radiologically proven early hip joint degeneration, which was symptomatic, and was excluded from the study group, leaving 35 subjects (all men, age 21.5 (3.8), height 184.9 (6.6) cm, weight 85.5 (7.1) kg, 25 right footers, 10 left footers; means (SD)). Four players had a past history of unilateral hernia repair (two during their football career and two as children) but were included in the study. Some players had a suspected or proven diagnosis of osteitis pubis or adductor tendonitis but were included in the study, as it was considered possible for these diagnoses to coexist with inguinal canal pathology.

All subjects were questioned about any recent history of groin pain on either side and had medical files examined. A player was determined as having suffered recent groin pain if he had had pain (in either lower abdominal or upper thigh region) over the
Accepted for publication 1 October 1997 


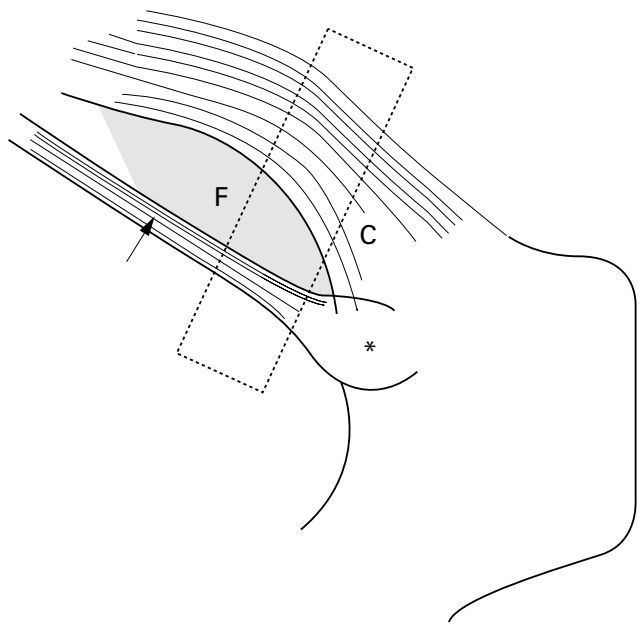

Figure 1 Transducer position (indicated by dashed lines). $A$ short axis view of the inguinal canal is obtained just lateral to the pubic tubercle $\left({ }^{\star}\right)$. Both conjoint tendon $(C)$ and transversalis fascia $(F)$ contribute to the posterior inguinal wall at this level. Arrow indicates inguinal ligament.

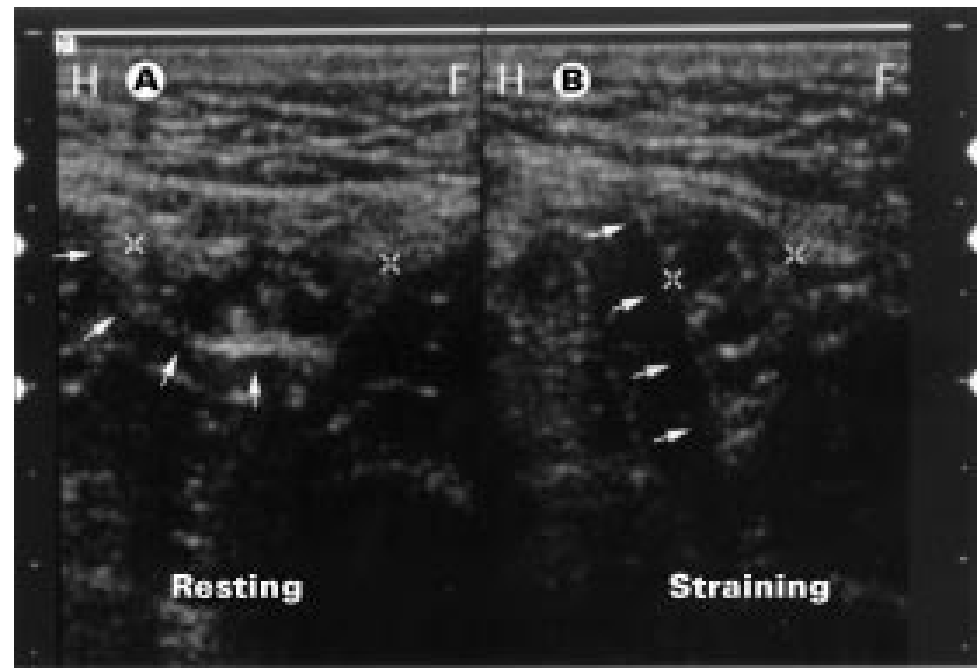

Figure 2 Short axis view of the normal inguinal canal at the level of superficial ring (the patient's head is to the left of the image). (A) At rest the posterior inguinal wall (arrows) is concave anteriorly and the spermatic cord is ovoid in shape. (B) Upon straining the posterior inguinal wall (arrows) straightens in a caudal direction. There is simultaneous reduction in the craniocaudal diameter of the spermatic cord.

previous eight months which had caused him to miss matches or had affected his match or training performance for a duration of longer than one month. For statistical analysis, the subjects were divided into two groups (groin pain and no groin pain), and variables were compared using $t$ tests and $\chi^{2}$ tests. Confidence intervals were determined using a Taylor series expansion.

Approval for the study was obtained from the University of New South Wales ethics committee.

\section{ULTRASOUND TECHNIQUE}

Players were examined towards the end of the 1995 football season. All ultrasound studies were performed by the same radiologist (JWR), who was unaware of the injury history of the players, using a $7.5 \mathrm{MHz}$ linear array transducer with the patient supine. Short axis real time views of each inguinal canal at the level of the superficial ring (transducer just lateral to the pubic tubercle (fig 1)) were chosen to permit a continuous or "dynamic" assessment of inguinal wall movement. Care was taken not to compress or distort the canal with excessive transducer pressure. In this position the resting inguinal canal showed an ovoid configuration (fig 2A). Motion of the inguinal canal and its walls was then observed as the patient was asked to strain several times using a variety of manoeuvres. Commands included: "tighten your tummy muscles hard", "push your tummy outwards", and "take a deep breath in and then strain down hard". A normal inguinal canal was diagnosed if, under stress, there was some degree of canal "closure" (a variable, sometimes only minimal, decrease in craniocaudal diameter and cross sectional area) (fig 2B). Posterior inguinal canal deficiency was diagnosed if, under stress, there was a dynamic increase in canal cross sectional area ("ballooning") (fig 3B). A convex anterior bulge of the posterior inguinal wall during stress (fig 3B) was usually also seen in association with ballooning of the canal, but was not used as an essential diagnostic feature at the time of this study as a discrete wall echo was occasionally difficult to distinguish clearly from adjacent tissues during brisk motion. Players were not evaluated by herniography. Some symptomatic players had other investigations including $x$ rays and bone scans.

\section{SURGICAL TECHNIOUE}

Some players who had significant chronic pain and were positive bilaterally on ultrasound examination subsequently chose to undergo surgical hernia repair. These were generally performed at the end of the season. All repairs were performed with open surgery using a variation of the Bassini technique. The repair of the posterior wall direct hernia was made with a 2.0 vicryl then 1.0 nylon suture without any prosthetic material. Where there was a deficiency of the conjoined tendon or other pathology seen, this was simultaneously repaired.

\section{Results}

Of the 35 players, 14 had a history of significant groin pain during the season. Six had predominantly right sided pain, three had mainly left sided pain, and five had bilateral pain. Only six players were forced to miss matches and therefore the other eight were hindered in match or training performance for longer than one month (to satisfy the study definition). There were a few other cases of players reporting minimal groin pain during the year, indicating a high general prevalence of groin pain in this population over the duration of one season.

On ultrasound examination of the inguinal regions, in 21 players $(60 \%)$ the abnormality of a dynamic insufficiency of the posterior wall was observed. Fifteen players $(43 \%)$ had this finding bilaterally, with six players having the abnormality on one side only (three left and three right). Overall, this yielded a rate of 36 deficient posterior walls from 70 canals examined. In 33 of the 36 reports, the ballooning 


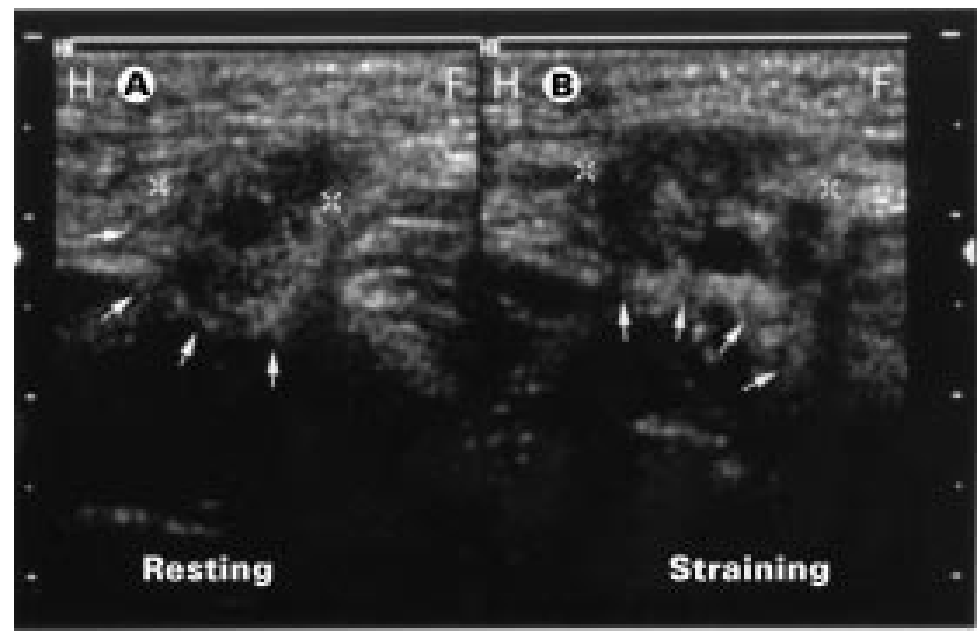

Figure 3 Ultrasound appearance of sports hernia. Short axis views of the inguinal canal at the level of superficial ring (the patient's head is to the left of the image). (A) At rest the posterior inguinal wall (arrows) shows minimal loss of normal smooth concavity. (B) Upon straining the posterior inguinal wall (arrows) shows a convex anterior bulge. The spermatic cord simultaneously increases in craniocaudal diameter, or "balloons", and displaces anteriorly. Static images, as shown here, are relatively subtle and do not convey the more impressive dynamics that are apparent on real time examination.

Table 1 Correlation of ultrasound findings with clinical symptoms

\begin{tabular}{lcc}
\hline & Groin pain & No pain \\
\hline Bilateral canal deficiency & 10 & 5 \\
At least one canal normal & 4 & 16 \\
\hline
\end{tabular}

was seen in the medial side of the canal, suggesting an incipient direct hernia, whilst in only three canals, the ballooning was seen more laterally and thought to be of the indirect variety. No players had evidence of a macroscopic direct or indirect hernia on examination or complained of any lump in the groin.

There was a strong association between the presence of bilateral inguinal canal deficiency and significant groin pain (table 1). This yielded a $\chi^{2}$ value of $7.78(\mathrm{df}=1, \mathrm{p}<0.01)$. Overall, there was no apparent correlation between the side of inguinal canal weakness and the side of pain, but this analysis was clouded by the large number of cases of bilateral abnormalities (there were only six unilateral cases). Subjects with bilateral deficiency were eight times more likely to have recent groin pain than those without $(95 \%$ confidence interval 1.73 to 37.1 ).

There was also a strong association between age and significant groin pain, with older players much more likely to suffer pain $\left(t_{33}=-3.23\right.$, $\mathrm{p}<0.01)$. Height, weight, and preferred kicking foot did not have any association with groin pain in this series. There was only a weak association between age and the presence of bilateral inguinal canal insufficiency $\left(t_{33}=1.69\right.$, $\mathrm{p}=0.15$ ).

To test whether age and bilateral canal deficiency were independent risk factors, logistic regression was performed to predict significant pain on the basis of whether or not the player had bilateral inguinal canal insufficiency and whether or not the player was over 22 years of age. Both variables ("age $>22$ " and "bilateral positive scan") were entered into the equation, which had a combined $\chi^{2}$ value of 16.49 $(\mathrm{p}<0.001)$. This was more significant than either variable considered separately.

The four players who had undergone previous (all unilateral) hernia repairs did not have any deficiency on the side of the repair, with one player being positive on the other side.

One symptomatic player had recently been diagnosed as having osteitis pubis, based on typical radiographic and bone scan findings. Four players defined as having significant groin pain (two who had a positive ultrasound and two who were negative) were considered to have a diagnosis of adductor tendonitis, based on local tenderness at the adductor origin and improvement after a local anaesthetic and cortisone injection to this area.

FOLLOW UP

Nine players who had both significant groin pain and were positive bilaterally for canal insufficiency and who were referred for specialist surgical opinion proceeded to have hernia surgery. One player was operated on during the season for debilitating pain, but all other players were able to wait until the end of the season. Seven players had bilateral repairs, with one left sided and one right sided operation performed. Surgical findings correlated well with ultrasound diagnosis, with the major finding at operation being a weak bulging posterior wall of the inguinal canal. There was one finding of lipoma of the cord at surgery which had been reported as an indirect hernia on ultrasound.

Five of the nine players had results that were considered to be good or excellent, after at least 12 months of follow up, with substantial improvement of symptoms. Where mild symptoms still persisted, these were offering no impediment to football competition or training.

Two players had significant residual pain after surgery and were considered to have poor outcomes. Both had had a bilateral hernia repair and had ongoing pain in the lower groin (adductor region). One felt 50\% improved at 11 weeks after the operation but his residual pain had definitely localised to the right adductor tendon. He underwent right adductor tenotomy at this stage and returned to play with fewer symptoms. The other had no improvement from the hernia repairs and subsequently had a left obturator nerve release, which significantly improved his symptoms.

Two players were considered to have fair results from the surgery. One had a good response to the initial repair, with pain fully resolved for six months. However, he had a sudden onset (on the same side as his initial symptoms) of adductor pain six months later which was found to be an adductor tendon tear seen on ultrasound. The last player had a good initial result from the surgery but his original symptoms returned to a milder extent six months later. His follow up ultrasound showed that the bilateral ballooning had returned.

The player with osteitis pubis had an excellent outcome with bilateral hernia repair and was able to return to competitive football seven weeks after surgery; this condition has a 
typical time of nine months to recovery with conservative treatment. ${ }^{13}$

Of interest was a further player included in this study as a false positive result. $\mathrm{He}$ had bilateral ballooning on straining but no history of significant groin pain. In the season subsequent to the initial scan, he developed mild groin pain associated with a macroscopic (clinically apparent) right inguinal hernia, which required surgery.

Six of the players who had undergone hernia repair(s) were examined by ultrasound two to six months after surgery. All six had improved symptoms but only two had totally normal findings using the study criteria. Four of the six players still exhibited ballooning of one inguinal canal on straining, but in all cases it was comparably of a lesser degree than before surgery.

\section{Discussion}

Hernia surgery for cases of chronic debilitating groin pain in athletes with no sign of clinical hernia was controversial when first performed 10-15 years ago and still arouses some scepticism. ${ }^{24}$ Good or excellent surgical results have consistently been reported from Scandinavia, ${ }^{1617}$ Australia,${ }^{14}{ }^{15}$ the United Kingdom, ${ }^{12}{ }^{25}$ the United States, ${ }^{11}{ }^{26}$ and continental Europe 2728 which have made this operation a part of standard sports medicine practice.

Hernia repair for clinically evident hernia is one of the most common procedures in general surgery. The pathophysiology of direct hernia, which is most commonly found in older men, is a deficient posterior wall. Anatomists have found this abnormality to exist in a very high proportion $(25 \%)$ of the general male population, ${ }^{29}{ }^{30}$ who mostly do not present with clinical hernia.

Despite the good results of surgery and a growing number of surgeons willing to operate on athletes with a typical history of this condition, the actual source of pain and best methods for diagnosis remain unclear. The gold standard for diagnosis of the condition is considered to be an observation of posterior inguinal wall laxity at surgical exploration, ${ }^{7}$ which is predictably seen by most surgeons reporting their cases and does not help in the decision as to whether an operation is indicated. Clinical findings include a history of chronic poorly localised groin pain which is aggravated by running, kicking, and performing sit ups. Sometimes the pain is well localised with tenderness over the conjoint tendon, but it is quite often difficult to differentiate between inguinal canal pathology and other causes of groin pain. With respect to the examination of the inguinal canal, a bulge or prominence that is apparent on standing is diagnostic of pathology, but a prominence felt on coughing or straining only is often present in asymptomatic men.

This paper reports clinical and surgical correlations of a technique for ultrasound diagnosis of incipient inguinal hernia in athletes which are similar to the results obtained by herniography. The underlying abnormality in these cases is an insufficiency of the posterior inguinal wall at the level of the superficial ring that appears as an anterior bulge on straining and causes "ballooning" of the inguinal canal. The "normal" canal does not balloon and usually exhibits caudal tilt as the conjoint tendon tightens and descends (the so-called shutter mechanism). However, ballooning alone should not be regarded as diagnostic for inguinal hernia, as this ultrasound finding can also be caused by varicocele or lipoma of the cord. Therefore careful attention must also be paid to the pattern of posterior inguinal wall motion, as anterior displacement and a progressive convex anterior bulge of the posterior inguinal wall under stress are important features that serve to increase the specificity of a positive report. An indirect inguinal hernia that extends to the level of superficial inguinal ring on straining can also cause ballooning, and routine assessment of the deep inguinal ring is therefore also recommended.

A number of factors suggest that the ultrasound diagnosis of hernia may be highly operator dependent. These include the fact that most radiologists would find the sonographic anatomy of the inguinal region initially unfamiliar and confusing, the great variation of ultrasound image quality with body habitus, and the need for a minimum level of experience in evaluating inguinal wall motion. Furthermore, differences might be expected in observer opinion, scan technique, and patient cooperation. Correct transducer position at the level of superficial inguinal ring is particularly important, and the difficulty that sometimes exists in deciding between normality and borderline abnormality should also be recognised.

In this study there were a significant number of positive results in asymptomatic athletes and the high number of bilaterally positive tests. These findings are also noted in studies of herniography, ${ }^{717}$ and have been thought to be "false positive" investigations. Given that the same "false positives" are seen with two different imaging modalities, it is possible that the positive tests represent significant findings that are precursor lesions to the clinical syndrome of groin pain in susceptible athletes. Another relevant finding of the current study is the strong correlation between age and significant groin pain, which was independent of canal deficiency. These findings support a hypothesis that the syndrome of athlete's hernia is caused by a combination of an aggravating activity plus a structural susceptibility plus time (repetitive strain).

The sample size in this study was large enough to show a statistically significant correlation between bilateral canal deficiency and groin pain but with a wide confidence interval. Because this was not a prospective study, whether the inguinal canal insufficiency preceded the development of groin pain in the symptomatic cases cannot be determined. It does not resolve the question of whether the ultrasound findings represent a normal variation which is more susceptible to groin pathology, a precursor lesion to a pathological entity 
("sports hernia") or even a sequela of groin pain from any cause (which has been suggested by Fredberg and Kissmeyer-Nielsen ${ }^{24}$ ).

Of further interest is the failure of many of the subjects who underwent hernia repair to return to "normal" ultrasound findings, despite clinical improvement. Hernia repair in the general population has a significant failure rate, estimated to be $10 \% .^{31}$ Previous reports of incipient hernias in athletes have focused on diagnosis and implied that if surgery is performed then the condition will be cured. It is likely that there is a surgical failure rate in the athletic population (in addition to diagnostic errors). The best type of repair in athletes is not established, but comparison of pre- and post-operative ultrasound offers an excellent method to research this further.

Posterior wall deficiency can coexist with other groin pathology. It should be considered as a potential factor in the pathophysiology of osteitis pubis. Although many references consider that osteitis pubis has a poor prognosis and advocate conservative treatment, some authors recommend hernia repair for this condition. ${ }^{217}$ Adductor and psoas tendonitis, stress fractures, referred pain from the sacroiliac joint, and lumbar spine and nerve entrapments $^{32}$ are some of a large range of other conditions that can have a similar presentation. When considering groin pain in an athlete, hip joint range of motion should be assessed, as the incidence of hip joint chondral pathology is probably underestimated. Referral to a sports physician with an interest in this area is indicated in a case of chronic groin pain that is proving difficult to diagnose.

In some presentations of medial groin pain, clinical examination can localise the pathology to the suprapubic (abdominal tendons/ inguinal canal) or subpubic (adductor tendons) area. However, when the pain arising from either area has become chronic, there is often associated pubic symphysis degeneration. This results in tenderness on both the superior and inferior margins of the pubic symphysis and pain on both resisted hip adduction and abdominal muscle contraction-for example, sit ups. It is these cases in which investigations are required to differentiate abdominal from adductor insertion pathology. Ultrasound has previously been shown to also be of value in diagnosing adductor tendon pathology. ${ }^{33}$ Both technetium bone scan and magnetic resonance imaging studies have potential to show and differentiate pathology in the abdominal and adductor regions. Although the syndrome of sports hernia may not present with macroscopic hernia on clinical examination, it is recommended that other objective criteria are met before surgical exploration is attempted. These should include chronic pain on movements of the abdominal musculature (including kicking, running, sit ups, coughing, straining) and radiological evidence of pathology in this area (positive ultrasound as described, positive herniogram, or bone scan with increased uptake along superior pubic ramus).
This study suggests that the mechanics of the inguinal canal are significant in the development of groin pain in elite Australian footballers and confirms previous reports that the primary abnormality in sports hernia is a deficiency of the posterior wall of the canal. It is not certain whether this lesion causes pain through a primary mechanism - that is, pain is felt because of herniation of peritoneum on straining - or through secondary conditionsfor example, enthesopathy of abdominal muscle tendon insertions. Based on literature reports of this condition, it most commonly occurs in sports that involve repetitive kicking-for example, soccer and Australian football. In other sports where there is minimal kicking and the mechanism of injury is mainly through repetitive rapid changing of direction-for example, rugby football, ice hockey - the adductor muscle group appears to be a more common source of groin pain. This area remains somewhat of an enigma, with multiple pathologies often coexisting.

1 Seward H, Orchard J, Hazard H, Collinson D. Football njuries in Australia at the elite level. Med $\mathcal{f}$ Aust 1993;159:298-301.

2 Xethalis JL, Lorei MP. Soccer injuries. In Nicholas JA, Hershman EB, eds. The Lower extremity and spine in sports medicine. St. Louis: Mosby, 1995:1519-25.

3 Gibbs N. Common rugby league injuries. Sports Med 1994; 18:438-50.

4 Brukner P, Khan K. Clinical sports medicine. Sydney: McGraw-Hill, 1993:314.

5 Ekberg O, Persson N, Abrahamsson P, et al. Longstanding groin pain in athletes: a multidisciplinary approach. Sports Med 1988;6:56-61.

6 Estwanick JJ, Sloane B, Rosenberg MA. Groin strain and other possible causes of groin pain. Physician Sportsmedicine 1990;18:54-65.

7 Lovell G. The diagnosis of chronic groin pain in athletes: a review of 189 cases. Aust F Sci Med Sport 1995;27:76-9.

8 Zimmerman G. Groin pain in athletes. Aust Fam Physician 1988;17:1046-52.

9 Hackney RG. The sports hernia: a cause of chronic groin pain. Br $\mathcal{F}$ Sports Med 1993;27:58-62.

10 Crichton KJ, Fricker PA, Purdam C, Watson AS. Injuries to the pelvis and lower limb. In Bloomfield J, Fricker PA, Fitch $\mathrm{KD}$, eds. Textbook of science and medicine in sport. Melbourne: Blackwell, 1995:432-34.

11 Taylor DC, Meyers WC, Moylan JA, et al. Abdominal musculature abnormalities as a cause of groin pain in athletes: inguinal hernias and pubalgia. Am f Sports Med 1991;19: 239-42.

12 Gilmore OJA. Gilmore's groin. Sportsmedicine and Soft Tissue Trauma 1992;3.

13 Fricker PA, Taunton JE, Ammann W. Osteitis pubis in athletes: infection, inflammation or injury? Sports Med 991;12:266-79.

14 Malycha P, Lovell G. Inguinal surgery in athletes with chronic groin pain: the sportsman's hernia. Aust N Z F Surg 1992;62:123-5.

15 Polglase AL, Frydman GM, Farmer KC. Inguinal surgery or debilitating chronic groin pain in athletes. Med $\mathcal{F}$ Aust 1991;155:674-7.

16 Ekberg O, Blomquist P, Olsson S. Positive contrast herniography in adult patients with obscure groin pain. Surgery 1981;89:532-5.

17 Smedberg SGG, Broome AEA, Gullmo A, Roos H. Herniography in athletes with groin pain. Am f Surg 1985;149: 378-82.

18 Ekberg O. Complications after herniography in adults. $A m \mathcal{F}$ Radiol 1983;140:491-5.

19 Schumpelick V, Treutner KH, Arlt G. Inguinal hernia repair in adults. Lancet 1994;344:375-9.

20 Harrison LA, Keesling CA, Martin NL, et al. Abdominal wall hernias: review of herniography and correlation with cross-sectional imaging. Radiographics 1995;15:315-32.

1 Lawrenz K, Hollman AS, Carachi R, Cacciaguerra S. Ultrasound assessment of the contralateral groin in infants with unilateral inguinal hernia. Clin Radiol 1994;49:546-8.

22 Truong S, Pfingsten FP, Dreuw B, Schumpelick V. Value of sonography in diagnosis of uncertain lesions of the abdominal wall and inguinal region. Chirurg 1993;64:468-75.

23 Arregui ME. The value of ultrasound in the diagnosis of hernias. In: Arregui ME, Nagan RF, eds. Inguinal hernia. Advances or controversies? Oxford: Radcliffe, 1994:73-9.

24 Fredberg U, Kissmeyer-Nielsen P. The sportsman's hernia - fact or fiction? Scand f Med Sci Sports 1996;6:201-4.

25 Urquart DS, Packer GJ, McLatchie GR. Return to sport and patient satisfaction levels after surgical treatment for groin disruption. Sports Exercise and Injury 1996;2:37-42. 
26 Simonet WT, Saylor HL, Sim L. Abdominal wall muscle tears in hockey players. Int F Sports Med 1995;16:126-8.

27 Christel P, Djian P, Roger B, et al. Apport de l'IRM dans la strategie du traitement chirurgical des pubalgies. Fournal de Traumatologie du Sport 1996;13:95-101.

28 Rochcongar P, Le Gall F, Jan J. La pubalgie du sportif: mise au point a propos d'une etude retrospective de 214 patients. Science $\mathcal{E}$ Sports 1996;11:135-9.

29 Scott P. External herniae. In: Dudley H, Waxman B, eds. $A n$ aid to clinical surgery, 3rd ed. Edinburgh: Churchill Livingstone, 1984:231.
30 Skandalakis JE, Gray SW, Skandalakis LJ, et al. Surgical anatomy of the inguinal area. World $\mathcal{F}$ Surg 1989;13:490-8.
Lichtenstein IL, Shulman AG, Amid PK. The cause, prevention and treatment of recurrent groin hernia. Surg prevention and treatment of recur
Clin North Am 1993;73:529-44.

32 Bradshaw C, McCrory P, Bell S, Brukner P. Obturator nerve entrapment: a cause of groin pain in athletes. Am $\mathcal{F}$ Sports Med 1997;25:402-8

33 Kalebo P, Karlsson, J, Sward L, Peterson L. Ultrasonography of chronic tendon injuries in the groin. Am $\mathcal{F}$ Sports Med 1992;20:634-9.

\section{Commentary}

This paper adds to the growing literature supporting the concept of a weak posterior inguinal wall causing groin pain in sportsmen and women. The diagnosis is difficult to confirm and is largely dependent upon the skill of the examining clinician. There remains a great deal of scepticism outside the sports medicine fraternity. An objective test is desirable. In my experience, herniography is not sufficiently sensitive to detect the early smaller sport hernias and requires an experienced radiologist. Ultrasound, from the evidence presented here, may be sufficiently sensitive to be able to pick up hernias before they become symptomatic. We may come to rely upon the skills of the examining radiologist!

R G HACKNEY

\section{Fracture healing: the enigma}

Millions of British football fans breathed a sigh of relief at Alan Shearer's return for the World Cup, fully recovered from an ankle fracture. However, be it superstar or novice the surgeon is always faced with the dilemma, "When is the fracture united?". Most sports fractures are fixed internally with screws and plates, and thus opposition is accurate, joint surfaces realigned, bone length restored, and soft tissue damage, especially to ligaments, repaired. The latter is as important as the former! But firm fixation means little callus, the fracture cannot be stressed clinically to determine "give", and specialised scans with MRI or DEXA are inconclusive or impossible. Also, pain and swelling may arise from the devices catching the soft tissues and confuse the issue, while to play with screws and plates in situ risks refracture at the stress interface between metal and bone. At best the fixation can be removed after three months when direct union is proceeding, and the fractures further stressed by controlled weight bearing exercises. Full activities may be encouraged from the fifth month if all is progressing well-otherwise, "caution is the name of the game". Generally, players are match fit by nine months. At that time there should be no pain or swelling, no effusion, and no restriction of ankle movement, including inversion and eversion. Jumping, shuttles, sprinting, and (most of all) kicking must have returned to the level before injury. Unlike the tibia, delayed or non-union is rare. So it's welcome back and good luck!

D S MUCKLE Middlesborough 